\title{
TOTAL ARTHROPLASTY OF THE HIP AFTER CHILDHOOD SEPSIS
}

\author{
YOUNG-HOO KIM
}

From the Texas Tech University Health Sciences Center, Lubbock, Texas

\begin{abstract}
Total hip arthroplasty was performed in $\mathbf{4 5}$ hips of 44 patients who had pyogenic arthritis of the hip during childhood. The average age of the patients was 36.4 years (range 19 to 50). The interval between active infection and arthroplasty ranged from 11 to $\mathbf{4 0}$ years, and average follow-up was 65.4 months (range 58 to 80).

Specimens of tissue taken before and during operation gave negative cultures in all hips, and no patient had reactivation of infection. The mean functional rating of the hips was 58 points before operation and 90 at the final review.

Complications were seen in 11 cases (24\%), loosening of components occurred in six (13\%) and one hip had to be revised for acetabular component migration.

Acetabular allografts were required in 27 hips (60\%). All allografts united but there was partial resorption of the graft in the non-weight-bearing area in all.
\end{abstract}

Patients with hypoplastic and dysplastic hips secondary to childhood pyogenic arthritis present a special surgical challenge for total hip arthroplasty, because of longstanding distortion of the bone and soft-tissue anatomy. The majority of patients are young and they are reported to have a high incidence of failure of component fixation (Chandler et al 1981; Dorr, Takei and Conaty 1983).

A dysplastic femoral canal limits the size and shape of the femoral component. Several authors have advocated the use of a small straight femoral stem after resection of the proximal femur at the level of, or distal to, the lesser trochanter (Charnley and Feagin 1973; Halley and Charnley 1975) although a high incidence of component loosening has been reported (Dunn and Hess 1976; Woolson and Harris 1983).

The purpose of this study is to review the results of total hip arthroplasty with a cementless porous-coated prosthesis.

\section{PATIENTS AND METHODS}

The study involved 44 patients ( 45 hips, 18 men and 26 women) treated at three hospitals in South Korea. Their average age was 36.4 years (range 19 to 50 ). The average

Y-H. Kim, MD, Associate Professor

Department of Orthopaedic Surgery, Texas Tech University Health Sciences Center, Lubbock, Texas 79430, USA.

(C) 1991 British Editorial Society of Bone and Joint Surgery

0301-620X/91/5166\$2.00

J Bone Joint Surg [ Br] 1991 ; 73-B:783-6. time interval between the resolution of active infection in the hip and arthroplasty was 19.9 years (range 11 to 40 ).

Investigations were carried out pre-operatively and intraoperatively for evidence of continuing infection. Pre-operative aspiration was attempted in all patients; if no fluid was obtained from the joint, saline washings were performed. Cultures from aspirates, intra-operative smears and excised specimens were studied for growth of aerobic, anaerobic, and tubercle bacilli, and histological preparations stained with haematoxylin and eosin were examined for evidence of active infection.

The usual clinical, haematological, and radiographic data were recorded for each patient as were reports of previous operations and relevant laboratory and clinical features, such as the presence of draining sinuses. Any radiolucent area that might represent localised osteolysis was especially noted in the planning of the procedure. During the operation, antibiotics were withheld until material from deep tissues had been obtained for culture, and then cephalosporin (daily dose $6 \mathrm{~g}$ ) was administered for two days intravenously.

Limb length discrepancy was measured before and after operation, using a tape measure and a scanogram.

Five different patterns of cementless prostheses were used. Twenty-three were porous-coated anatomic (PCA : Howmedica, Rutherford, NJ, USA), nine were anatomical medullary locking porocoat (AML: Depuy, Warsaw, IN, USA), seven were Harris-Galante porous (HGP: Zimmer, Warsaw, IN, USA), three were BIAS (Zimmer), and two were anatomical medullary locking acetabular 
components with Müller trilock femoral components (Dupuy). Allografts were used to help reconstruct the acetabulum in 27 hips $(60 \%)$.

The average duration of follow-up was 65.4 months (58 to 80$)$. The patients were evaluated at each stage using the Harris hip rating system (1969). At all followup visits, anteroposterior, frog lateral, and cross-table lateral radiographs were made of each hip. These were compared with the immediate postoperative films for radiolucent lines at the bone-implant interface. They were assessed by measuring the width of the lucent line in several zones. The femoral interface was divided into 14 zones (seven on the anteroposterior view and seven on the lateral) and the acetabular interface was divided into three equal zones.

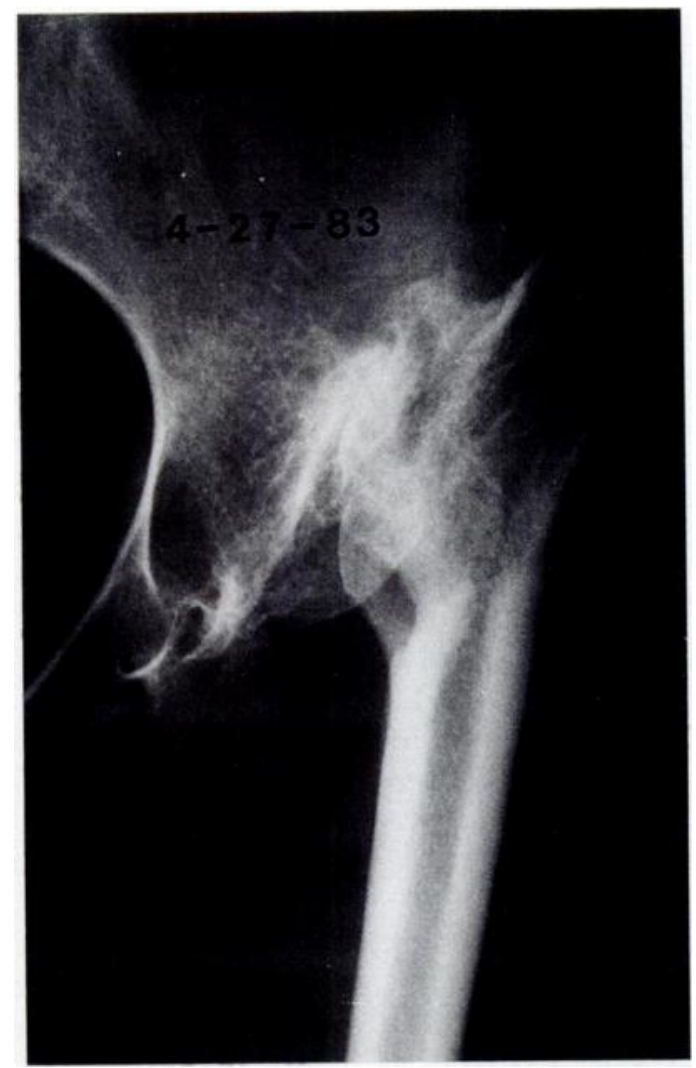

Fig. 1

Radiograph of the left hip of a man aged 28 years showing complete destruction of the femoral head and neck, a high-riding greater trochanter and severe acetabular dysplasia.

The following information about the femoral component was obtained from the first postoperative film: 1) the position of the femoral component within the intramedullary canal (valgus, neutral, or varus).

2) the dimensional ratio between the femoral component and the femoral canal in coronal and sagittal planes at the upper border of the lesser trochanter, at the middle of the stem and $1 \mathrm{~cm}$ proximal to the tip of the prosthesis. The stem- canal ratio at the first level indicated the degree of metaphyseal fill of the prosthesis. Evaluation at the latter two levels enabled us to assess whether the stem filled the distal canal adequately.

The position of the acetabular component was assessed from the postoperative films. Vertical migration was determined by measuring the change in the vertical distance between the inferior margin of the acetabular cup and the inferior margin of the ipsilateral teardrop; horizontal migration was measured as change in the horizontal distance between Köhler's line and the centre of the outer shell of the acetabular cup (Callaghan, Dysart and Savory 1988).

Loosening of the femoral component was diagnosed if there was progressive varus shift or progressive axial subsidence. The acetabular component was considered loose if there was a change in position or a continuous radiolucent line of more than $2 \mathrm{~mm}$ width.

\section{RESULTS}

No patient had recurrent infection or delayed wound healing and the sedimentation rate was always within the normal range. Laboratory investigations including

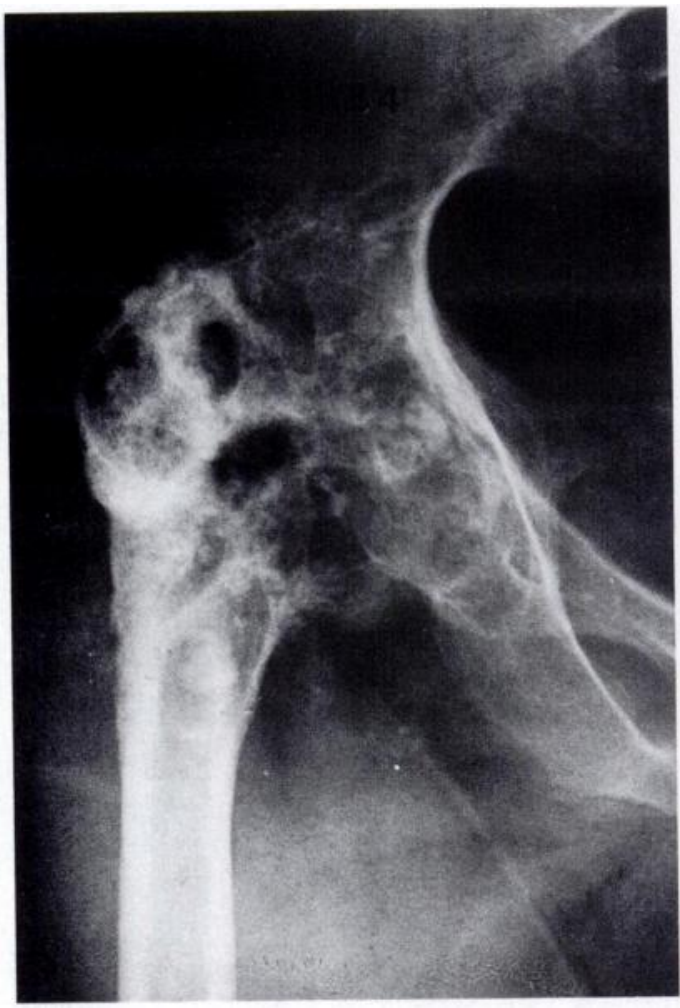

Fig. 2

Radiograph of the right hip of a man aged 37 years showing severe destruction of the hip, hypoplasia of the femur and acetabular dysplasia. 
blood count, serum chemistry, and urinalysis, were normal in all patients. All cultures of saline washings from pre-operative aspirations proved sterile. The smears of aspirates and biopsy specimens all showed less than five polymorphonuclear leucocytes per high power field and no bacteria.

Radiographs revealed complete destruction of the femoral head and neck with a high-riding greater trochanter and severe acetabular dysplasia in three hips (Fig. 1); solid fusion of the hip with a proximally migrated femur and dysplasia of the acetabulum in six ; and severe destruction of the hip with hypoplasia of the femur in thirty-six (Fig. 2). in the neutral position, one was in $10^{\circ}$ varus and one in $10^{\circ}$ valgus. In 40 of the 45 stems, the average percentage of the medullary canal filled by the prosthesis on the anteroposterior radiograph was $82 \%$ at the metaphysis, $84 \%$ at mid-stem and $73 \%$ at the tip. The equivalent measurements on the lateral radiographs were $70 \%, 73 \%$ and $67 \%$. Three of the remaining five stems had a tight fit in the coronal plane (average $84 \%$ ), but were a poor fit in the sagittal plane (under $70 \%$ ). The other two cases had a poor fit in both planes (under $80 \%$ in the coronal plane and under $70 \%$ in the sagittal plane). Two of the three stems with a poor fit in the sagittal plane later became loose. Both cases in which the stems were poorly

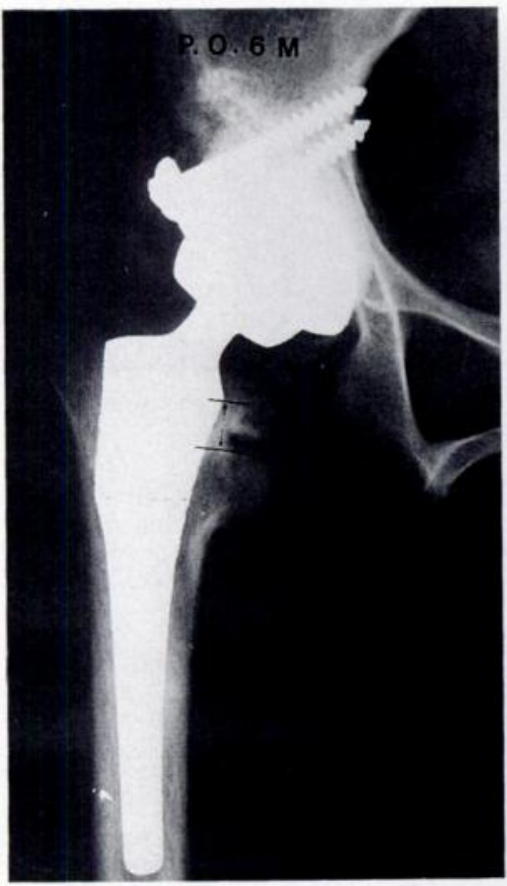

Fig. 3a

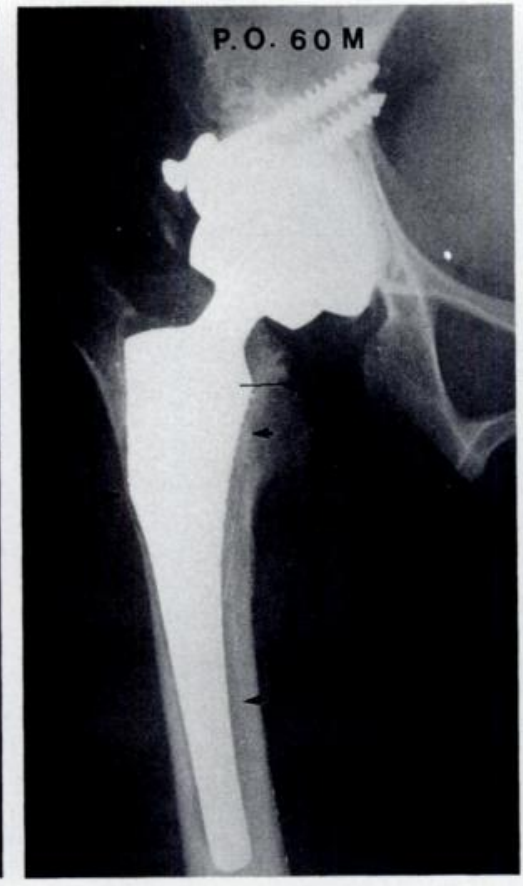

Fig. 3b

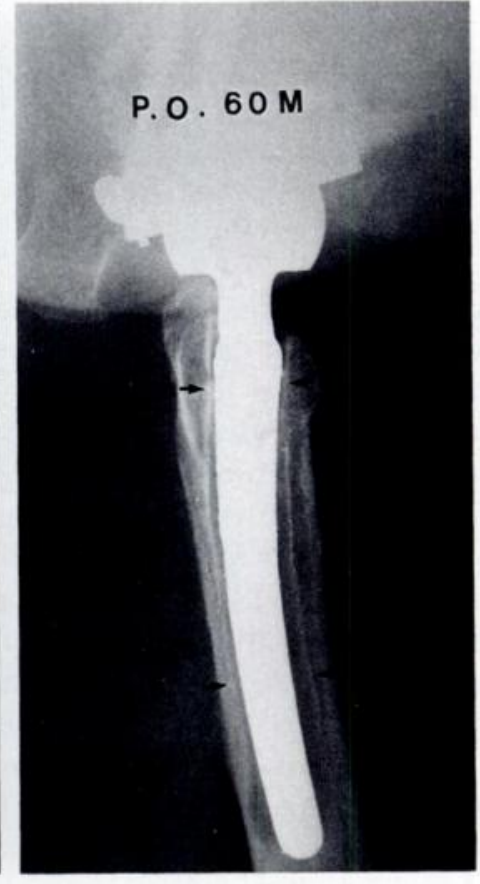

Fig. 3c

A series of radiographs of a 23-year-old woman with childhood pyogenic arthritis of the right hip. Figure 3a - An anteroposterior view taken six months postoperatively reveals that the PCA stem is placed in good position. Figure $3 \mathrm{~b}-$ The same view taken at 60 months demonstrating loosening and subsidence (about $10 \mathrm{~mm}$ on the femoral side). Figure $3 \mathrm{c}-\mathrm{A}$ lateral view of the same hip at 60 months reveals continuous radiolucent lines around the femoral stem wider than $2 \mathrm{~mm}$ in all zones.

Using the Harris hip rating system (1969), the mean pre-operative rating was 57.6 points $(7.5$ to 77$)$ and at the latest review was 90.4 points $(60$ to 100$)$. The average pre-operative flexion contracture was $5^{\circ}$ which improved to $2^{\circ}$. The average pre-operative range of flexion was approximately $75^{\circ}$ and improved to $102^{\circ}$ after operation. The pre-operative range of abduction, adduction, and internal and external rotation did not improve significantly after the arthroplasty. The pre-operative limblength discrepancy ranged from 0 to $7 \mathrm{~cm}$, with an average of $1.6 \mathrm{~cm}$; after operation the discrepancy was between 0 and $4 \mathrm{~cm}$ (average $0.9 \mathrm{~cm}$ ).

On the radiographs, 43 of the 45 femoral stems were fitting in both planes had secondary loosening of the femoral component (Figs 3a, b and c).

In the three completely dislocated high-riding hips, a BIAS stem was inserted after shortening the femur at the subtrochanteric level by 4 to $7 \mathrm{~cm}$ (Klisic and Jankovic 1976) and rotational stability was augmented with a plate and screws in two of these hips. At latest review of these cases, the clinical and radiographic results were excellent (Fig. 4).

The average inclination of the 45 acetabular components was $49.1^{\circ}$ from the horizontal $\left(26^{\circ}\right.$ to $\left.60^{\circ}\right)$. Thirtythree hips with PCA, AML, or HGP components had normal bone response without a radiolucent line, and ten 
hips showed a radiolucent line which was incomplete and narrower than $1 \mathrm{~mm}$. The remaining two hips, both with PCA prostheses, had a loose component.

All the acetabular allografts appeared to be well incorporated at the final follow-up. There was partial resorption of the allograft in the non-weight-bearing area in all, but the functional results were not compromised.

Complications. A high rate of complications was encountered in this series $(24 \%)$. There were six intra-operative and one postoperative femoral shaft fractures around the stem of the prosthesis. All were treated conservatively with a good result at the latest review. There were two instances of combined femoral and sciatic nerve palsy and one isolated sciatic palsy. These three patients had all had fused hips with proximally migrated femora and the nerves were probably stretched during mobilisation of the femur. All recovered completely. One hip developed grade IV heterotopic ossification (Brooker et al 1973).

\section{DISCUSSION}

In this series, there was no recurrence of infection in any hip, but all had been quiescent pre-operatively for more than ten years. Total hip arthroplasty appears to be a safe procedure after that length of time. This finding is consistent with our observations of total hip replacement for tuberculous arthritis (Kim, Han and Park 1987).

Four of the five hips with poorly fitting stems in one or both planes showed secondary component loosening with clinical symptoms. This finding supports the general opinion that initial security of cementless stems is important in both the sagittal and the coronal planes.

The outcome of the acetabular components has been encouraging in this series. In the hips which did not require allografts, the press-fit achieved with either pegs, spikes or screws provided adequate stability. However, if an acetabulum needs an allograft, it is recommended that two or three central screws are used to achieve fixation of the acetabular component.

Because of the extensive fibrosis of soft tissues from the previous infection, the patients did not obtain as good a range of motion as is usual after hip arthroplasty. Nevertheless, the majority did achieve some functional improvement.

Difficulty was often encountered fitting the stem in the dysplastic femoral canal, which frequently fractured. In the pre-operative planning, patients should have a CT scan to ensure that a prosthesis will fit in both planes and if not, a customised prosthesis should be made.

The author thanks Vana E. M. Kim, EdD, for reviewing and editing this manuscript, and Drs Dee-Yong Han, Ki-Soo Kim, Yup Kim, Jeong-Hwan Oh, and Seung-Hwan Oh, who referred the patients to me.

No benefits in any form have been received or will be received from a commercial party related directly or indirectly to the subject of this article.

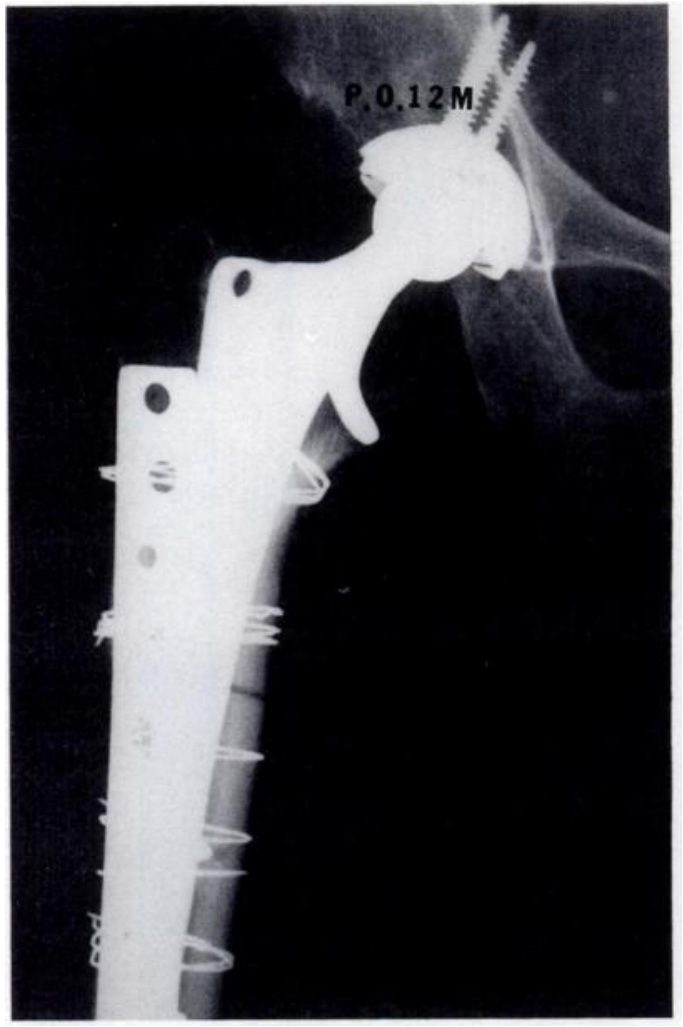

Fig. 4

Anteroposterior radiograph of a right hip 12 months postoperatively. The BIAS stem is partially obscured by an onlay plate which provides rotational security. The acetabular component is seated in good position.

\section{REFERENCES}

Brooker AF, Bowerman JW, Robinson RA, Riley LH Jr. Ectopic ossification following total hip replacement: incidence and a method of classification. J Bone Joint Surg [Am] 1973; 55-A: 1629-32.

Callaghan JJ, Dysart SH, Savory CG. The uncemented porous-coated anatomic total hip prosthesis: two-year result of a prospecive consecutive series. J Bone Joint Surg [Am] 1988; 70-A :337-46.

Chandler HP, Reineck FT, Wixson RL, McCarthy JC. Total hip replacement in patients younger than thirty years old: a five-year follow-up study. J Bone Joint Surg [ Am] 1981; 63-A :1426-34.

Charnley J, Feagin JA. Low-friction arthroplasty in congenital subluxation of the hip. Clin Orthop 1973; 91:98-113.

Dorr LD, Takei GK, Conaty JP. Total hip arthroplasties in patients less than forty-five years old. J Bone Joint Surg [Am] 1983; 65-A : 474-9.

Dunn HK, Hess WE. Total hip reconstruction in chronically dislocated hips. J Bone Joint Surg [ Am] 1976; 58-A :838-45.

Halley DK, Charnley J. Results of low friction arthroplasty in patients thirty years of age or younger. Clin Orthop 1975; 112:180-91.

Harris WH. Traumatic arthritis of the hip after dislocation and acetabular fractures: treatment by mold arthroplasty. J Bone Joint Surg [Am] 1969; 51-A :737-55.

Kim Y-H, Han D-Y, Park B-M. Total hip arthroplasty for tuberculous coxarthrosis. J Bone Joint Surg [ Am] 1987; 69-A:718-27.

Klisic P, Jankovic L. Combined procedure of open reduction and shortening of the femur in treatment of congenital dislocation of the hips in older children. Clin Orthop 1976; 119:60-9.

Woolson ST, Harris WH. Complex total hip replacement for dysplastic or hypoplastic hips using miniature or microminiature components. J Bone Joint Surg [ Am] 1983; 65-A :1099-108. 